\title{
Corrigendum
}

\section{Origin of endothelial progenitors in human postnatal bone marrow}

Morayma Reyes, Arkadiusz Dudek, Balkrishna Jahagirdar, Lisa Koodie, Paul H. Marker, and Catherine M. Verfaillie

Original citation: J. Clin. Invest. 109:337-346 (2002). doi:10.1172/JCI14327.

Citation for this corrigendum: J. Clin. Invest. 118:3813 (2008). doi:10.1172/JCI14327C1.

During the preparation of the manuscript, the FACS plot for $\beta 2$-microglobulin was erroneously duplicated to also represent CD62P in Figure 1B. The legend to Figure 5C was also affected by the error. The correct Figure 1B and legend to Figure 5C appear below.

Figure 1B
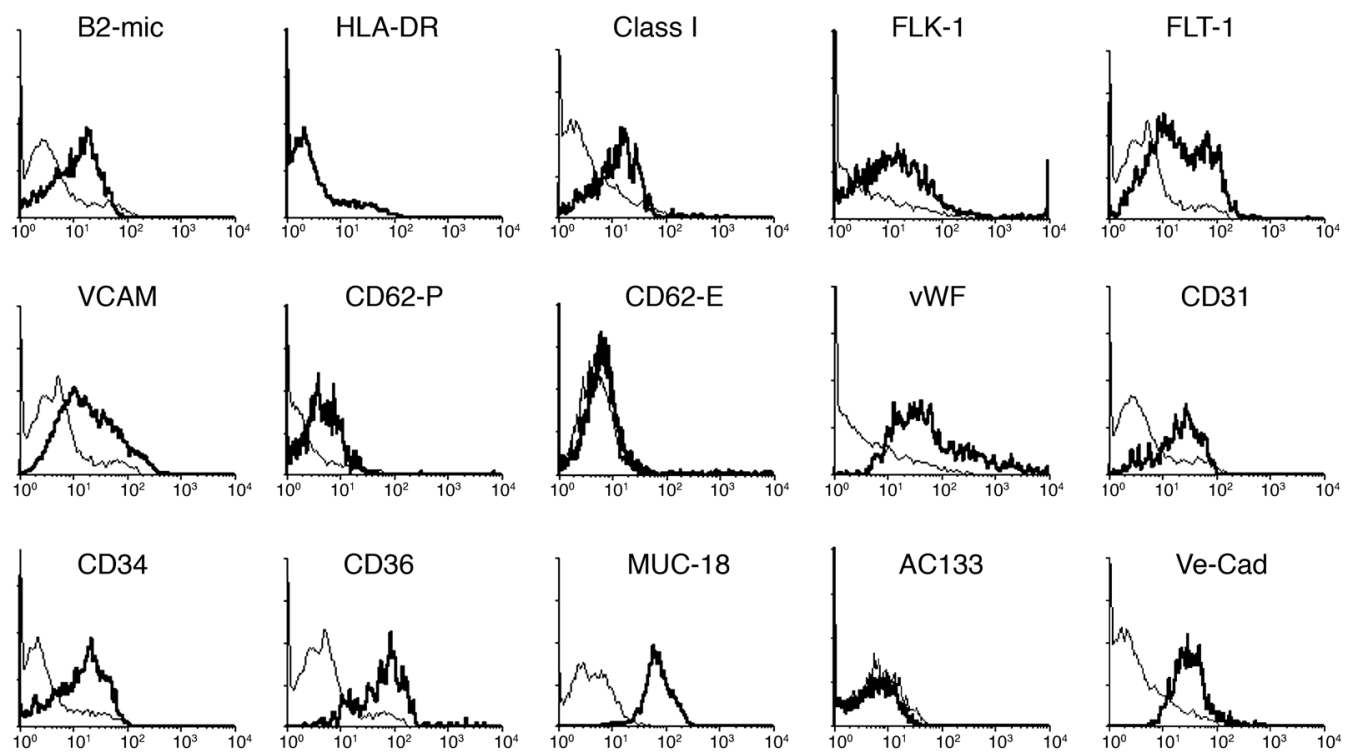

Figure 5C

IL-1 $\alpha$ induces expression of HLA-DR, a type of HLA class II antigen, and increases expression of adhesion receptors. MAPC-derived endothelial cells were incubated with or without $75 \mathrm{ng} / \mathrm{ml} \mathrm{IL-1} \alpha$ in serum-free medium for 24 hours. Cells were stained with Ab's against HLA class I, HLA class II, $\beta 2$-microglobulin, vWF, CD31, VCAM, CD62E, CD62P, or control Ab's and analyzed using FACS. Plots show isotype control IgG staining profile (thin line) versus specific Ab staining profile (thick line). Representative example of three experiments from three donors. Numbers above each plot show MFI for the control IgG staining and the specific Ab staining. Nl, normal; IL-1, induced populations. Note that the histograms for normal $\beta 2$-microglobulin, HLA class I, and HLA-DR are the same histograms presented in Figure 1B.

The authors regret the error. 